\title{
Wir durchlaufen alle eine exzentrische Bahn
}

\section{Friedrich Hölderlin und Johannes Kepler - ein Beitrag zum Hölderlin-Jahr 2020}

\author{
Hans-Joachim Albinus
}

\begin{abstract}
Hölderlin und Mathematik - wie passen sie zusammen? Und was sind die Beziehungen der beiden Geistesgrößen, einerseits der Dichter Hölderlin $(1770-1843)^{1}$ und andererseits der Astronom, Mathematiker und Physiker Kepler (1571-1630) ${ }^{2}$, die zwei Jahrhunderte trennen? Das scheint nur auf den ersten Blick verwunderlich, aber beider Lebenswege berührten gleiche Orte, gleiche Ausbildungsstätten und -inhalte (u. a. Mathematik und Astronomie), gleiche Gedankenwelten und Lebensentwürfe.
\end{abstract}

Ab Oktober 1788 wohnt Friedrich Hölderlin im Evangelischen Stift in Tübingen, um an der Universität das vorbereitende Magister-Artium-Grundstudium zu absolvieren, an das sich bestimmungsgemäß das eigentliche Theologiestudium anschließen soll.

Zuvor war er höherer Klosterschüler in Maulbronn und hatte dort über seine Jugendliebe Louise Nast (1768-1839) ihren Cousin Immanuel Gottlieb Nast (1769-1829) kennengelernt, Schreiber am Leonberger Rathaus, der sein enger Freund wurde. Nast und Hölderlin hatten einander in Maulbronn und Leonberg oft besucht. ${ }^{3}$

Der junge Hölderlin ist sich der Geschichtsträchtigkeit dieser drei Orte Maulbronn, Leonberg und Tübingen bewusst. Leonberg war der Wohnort Keplers Familie ab Ende 1575, hier war Johannes Kepler zuerst in die deutsche, dann in die lateinische Schule gegangen, bevor er mit herzoglichem Stipendium 1584 auf die niedere Klosterschule Adelberg und 1586 ebenfalls die höhere Klosterschule Maulbronn geschickt wurde ([1]). Viele Geistesgrößen nach Johannes Kepler und vor Hölderlin hatten schon diese „Schwäbische Laufbahn“ über die Klosterschulen ins Tübinger Stift und an die dortige Universität absolviert und stets das verbindliche von der philosophischen Fakultät betreute Grundstudium durchlaufen $([8,19])$. Seine sieben Artes liberales umfassten neben den sprachlichen Fächern Grammatik, Dialektik, Rhetorik (Trivium) die mathematisch-realen Arithmetik, Geometrie, Musik und Astronomie (Quadrivi$u m$ ), die vor allem mathematisch-theoretisch betrieben wurde, und wurden mit dem Magister Artium abgeschlossen, der somit eine andere Bedeutung als heute hatte. Dies hatte sich seit Keplers Zeiten nur wenig geändert ${ }^{4}([9,10,27])$.

Dennoch, genauso wie Keplers Liebe zur Poesie und zu den antiken römischen und griechischen Schriftstellern in Maulbronn und Tübingen entzündet wurde 5 , geschieht es auch bei Hölderlin, der sich nun wie damals schon Kepler intensiv mit Mathematik und Astronomie beschäftigen muss. ${ }^{6}$ Beide sind durch die Verpflichtung des herzoglichen Stipendiums zum Theologen bestimmt ([6, S. 12]), darüber hinaus Kepler aus eigenem Antrieb, Hölderlin durch seine pietistisch-fromme Mutter. Beide werden es jedoch nicht, Hölderlin aus Widerwillen gegen den Pfarrersberuf, Kepler wegen lebenslangen Verdachts calvinistischer, antilutherischer Gesinnung.
1589 wurde Kepler in das Tübinger Stift aufgenommen, und bewundernd widmet Hölderlin ihm genau 200 Jahre später eine Ode: ${ }^{7}$

Keppler

Unter den Sternen ergehet sich

Mein Geist, die Gefilde des Uranus

Überhin schwebt er und sinnt; einsam ist

Und gewagt, ehernen Tritt heischet die Bahn.

Wandle mit Kraft, wie der Held, einher!

Erhebe die Miene! doch nicht zu stolz,

Denn es naht, siehe es naht, hoch herab

Vom Gefild, wo der Triumf jubelt, der Mann,

Welcher den Denker in Albion,

Den Späher des Himmels um Mitternacht,

Ins Gefild tiefern Beschauns leitete,

Und voran leuchtend sich wagt' ins Labyrinth,

Daß der erhabenen Themse Stolz

Im Geiste sich beugend vor seinem Grab

Ins Gefild würdiger'n Lohns nach ihm rief:

„Du begannst, Suevias Sohn, wo es dem Blik

Aller Jahrtausende schwindelte;

Und ha! ich vollende, was du begannst,

Denn voran leuchtetest du, Herrlicher!

Im Labyrinth, Stralen beschwurst du in die Nacht.

Möge verzehren des Lebens Mark

Die Flamm' in der Brust - ich ereile dich,

Ich vollend's! denn sie ist groß, ernst und groß,

Deine Bahn, höhnet des Golds, lohnet sich selbst."

Wonne Walhallas! und ihn gebahr

Mein Vaterland? ihn, den die Themse pries?

Der zuerst ins Labyrinth Stralen schuf,

Und den Pfad, hin an dem Pol, wies dem Gestirn.

Heklas Gedonner vergäß' ich so, Und, gieng' ich auf Ottern, ich bebte nicht

In dem Stolz, daß er aus dir, Suevia!

Sich erhub, unser der Dank Albions ist. 


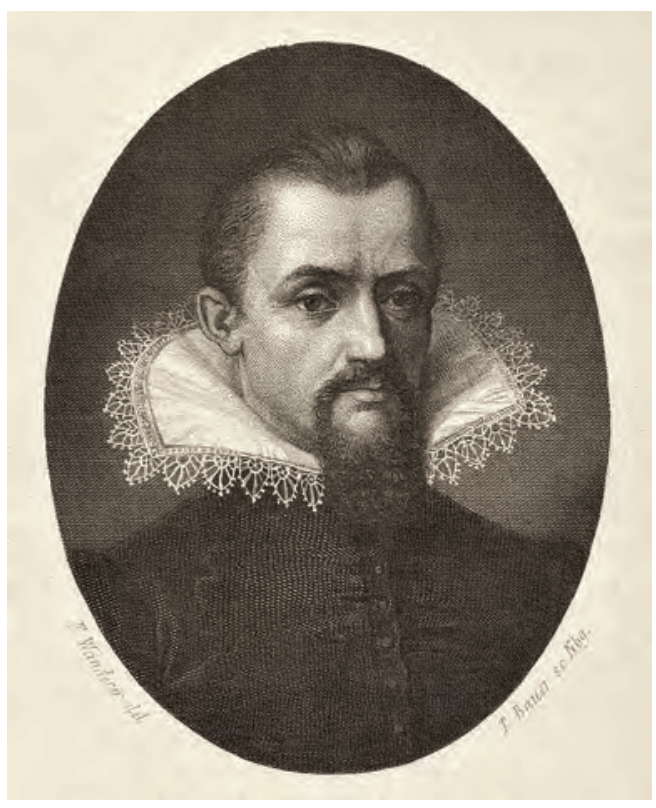

Johannes Kepler. Stich vor 1869 von Friedrich Wilhelm Wanderer (1840-1910), Maler, Zeichner, Illustrator und Professor an der Kunstgewerbeschule Nürnberg. (Foto: Hans Joachim Albinus)

Mutter der Redlichen! Suevia!

Du stille! dir jauchzen Aeonen zu

Du erzogst Männer des Lichts ohne Zal,

Des Geschlechts Mund, das da kommt, huldiget dir.

Hölderlin hat sich - angesteckt von der allgemeinen Verehrung der antiken Klassiker - ein besonders schwieriges Versund Strophenmaß ausgesucht, eine spezielle Odenform, die erst wenige Jahre zuvor Friedrich Gottlieb Klopstock (17241803) in die deutsche Dichtung eingeführt hat, unter viel Bewunderung, weil man die deutsche Sprache für die antiken Formen für ungeeignet hielt. Hölderlin wird nach Klopstock der zweite bedeutende Odendichter deutscher Sprache neben Friedrich Schiller (1759-1805) sein.

Thematisch ist das Gedicht interessant, zeigt es doch Hölderlins Beschäftigung mit Leben und Werk Keplers sowie mit Mathematik und Astronomie, die er noch weiter vertiefen will - er bedauert brieflich November 1791 gegenüber seinem Freund Christian Ludwig Neuffer (1769-1839) aus dem Tübinger Stift,

in hellen Nächten mich an Orion und Sirius, und dem Götterpaar Kastor und Pollux gewaidet ... Lieber! ich ärgre mich, daß ich nicht bälder auf die Astronomie gerathen bin. Diesen Winter soll's mein angelegentlichstes sein ([12, Bd. 6.1, S. 70-71]).

Auch in späteren Gedichten werden astronomische Motive noch eine Rolle spielen ([23]). Eine weitere Anregung wird das Gedenkstätten-Projekt Johann Jakob Atzels (1754-1816) in der von seinem Freund Schiller herausgegebenen Zeitschrift Wirtembergisches Repertorium der Litteratur (1782)

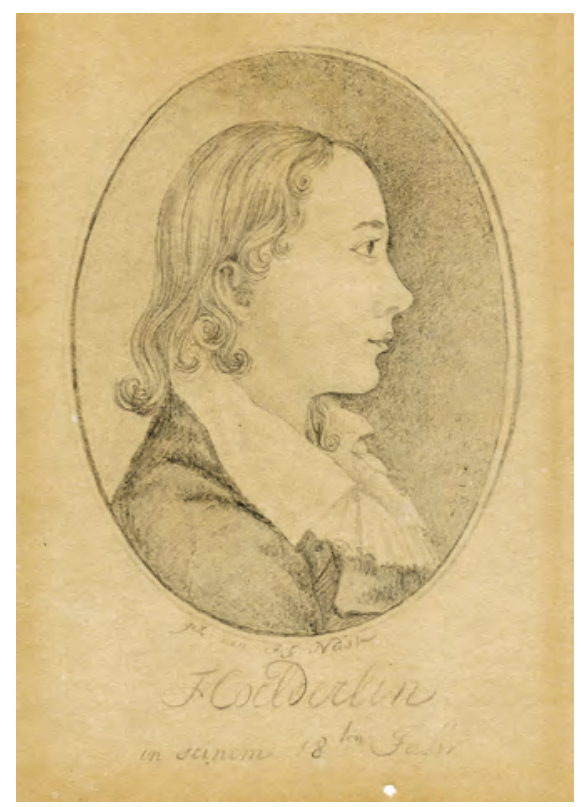

Friedrich Hölderlin mit 18 Jahren. Zeichnung von 1789 von Hölderlins Leonberger Freund Immanuel Gottlieb Nast, heute im Deutschen Literaturarchiv Marbach. (Foto: Deutsches Literaturarchiv Marbach)

gewesen sein, zu dem Schiller einige Inschriften für Denkmäler, neben dem Reformator Martin Luther (1483-1546) und Klopstock insbesondere auch für Kepler „in einer einsamen melancholischen Gegend“" beigesteuert hatte. ${ }^{8}$

Auch der Beginn der französischen Revolution 1789 ist ein Aspekt für die Entstehung des Gedichts, denn in Tübingen erhoffen sich Hölderlin und andere Gebildete enthusiastisch eine geistige Erneuerung der Verhältnisse in Gesellschaft und Politik, wie Kepler sie ehemals für die Astronomie eingeleitet hatte.

In des Uranus - erst 1781 von den Geschwistern Friedrich Wilhelm (1738-1822) und Caroline (1750-1848) Herschel entdeckt und zu Keplers Zeiten noch unbekannt - also äußerste und gefährlichste Bahn wagt sich Hölderlins Denken, so wie Kepler 1596 bis zum Saturn zur Erklärung seines ersten Weltmodells Mysterium Cosmographicum auf Basis der fünf mit ihren In- und Umkugeln ineinander geschachtelten Platonischen Körper ([1 7, Bd. 1]). Kepler überwindet es später selbst durch seine drei Gesetze der Planetenbewegung und bringt damit endgültig Licht in die Unkenntnis und das labyrinthische Dunkel aus falschen Theorien, der ptolemäischen, kopernikanischen und braheschen sowie seiner frühen eigenen, und weist damit den nachkommenden mitternächtlichen Beobachtern der Sterne den Weg. Er kennt jedoch die tiefere Ursache der Planetenbewegungen noch nicht, vermutet als astrophysikalische Begründung magnetische Kräfte, und erst Isaak Newton (1643-1727), der Denker in England (Albion), dem Land an der Themse, der in der 4.-6. Strophe zu uns und Kepler spricht, erkennt in der Gravitation die wahre Ursache, die die astronomischen Gesetze seines Vorgängers aus Schwaben (Suevia) vervollständigt und ihre Richtigkeit bestätigt, 9 ihn dadurch aber auch in Vergessenheit geraten lässt. 


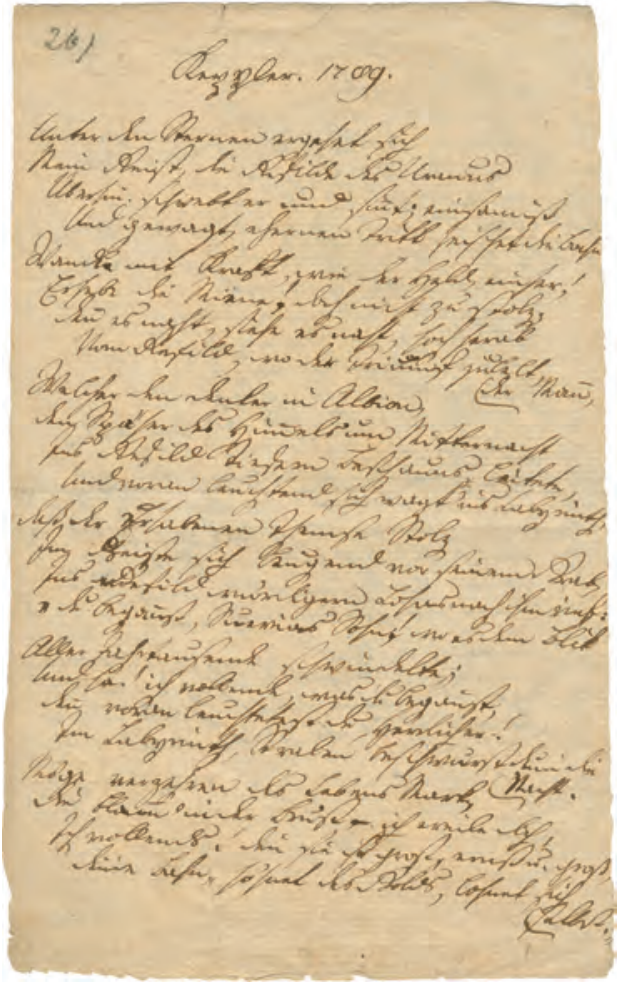

Hölderlins Manuskript Keppler, 1789, Seite 1. Heute im Deutschen Literaturarchiv Marbach. (Foto: Deutsches Literaturarchiv Marbach)

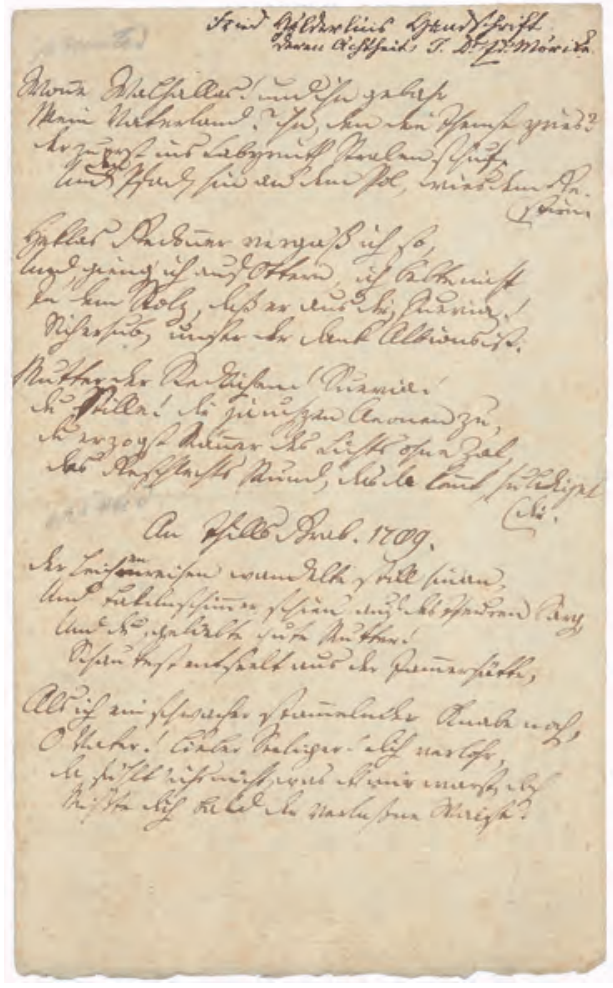

Hölderlins Manuskript Keppler, 1789, Seite 2. Rechts oben der Echtheitsvermerk Eduard Mörikes, unten die ersten beiden Strophen von An Thills Grab (ebenfalls 1789). Heute im Freien Deutschen Hochstift Frankfurt. (Foto: Freies Deutsches Hochstift Frankfurt)
In der sechsten Strophe - „Deine Bahn, höhnet des Golds, lohnet sich selbst" - wird Keplers ausstehende kaiserliche Besoldung angesprochen, ${ }^{10}$ ein Motiv, das die Romantik stilisiert bis zu Keplers Tod in völliger Mittellosigkeit. So dichtet Abraham Gotthelf Kästner (1719-180o), bedeutender Göttinger Mathematiker und Mathematikhistoriker und erster wissenschaftlicher Biograph Keplers, im vierten Band seiner Geschichte der Mathematik ([15, S. 387$])$ :

So hoch war noch kein Sterblicher gestiegen

Als Kepler stieg: Und starb in Hungersnoth!

Er wußte nur die Geister zu vergnügen,

Drum liessen ihn die Körper ohne Brot.

In den letzten drei Strophen schließlich scheint bereits die weitere Kepler-Rezeption des 19. Jahrhunderts auf. In der politischen und wirtschaftlichen Bedeutungslosigkeit der deutschen Kleinstaaterei sollten durch ihre Leistungen bedeutende Männer und Frauen das einigende Band eines deutschen Nationalstolzes bilden. Walhalla, die nordischmythologische Ruhestätte gefallener tapferer Kämpfer, soll nicht nur wie bei Hölderlin symbolisch-geistig bestehen, sondern wird 1842 als Gedenkstätte bedeutender Persönlichkeiten „deutscher Zunge“ mit Marmorbüsten und Gedenktafeln bei Regensburg als griechischer Tempel nach dem Vorbild des Parthenon in Athen erbaut. Kepler, der noch auf Grundlage klassischer geometrischer Methoden den Planeten ihre elliptischen Pfade um den ruhenden Pol der Sonne zuwies (1. Keplersches Gesetz) und die Verbindungsstrahlen Sonne $\leftrightarrow$ Planet mit der veränderlichen Umlaufgeschwindigkeit in Beziehung brachte (2. Keplersches Gesetz), wird jedenfalls in Form einer Büste ein Platz in der Walhalla zuteil. ${ }^{11}$

Der Stolz auf Keplers Leistung, die ihm Vorbild ist, und auf seine württembergische Bildung, die er in gleicher Weise durchläuft, lässt Hölderlin die Furcht vor eigenem Scheitern, versinnbildlicht durch das Tor zur Hölle, der Sage nach der Vulkan Hekla auf Island, und die Giftschlangen, die Ottern, vergessen. Hölderlin selbst sollte jedoch - anders als seinen literarischen Vorbildern Klopstock und Schiller - kein Platz in der Walhalla beschieden sein, er war der Nachwelt zu wenig Identifikationsgestalt, zu wenig heroisch, zu wenig geradlinig und seine zweite Lebenshälfte ab 1807 durch die unheilbare Krankheit im Tübinger Turmzimmer überschattet. Erst verspätet, wie zuvor schon bei Kepler, hat man seine Bedeutung gewürdigt.

Wir durchlaufen alle eine exzentrische Bahn, und es ist kein anderer Weg möglich von der Kindheit zur Vollendung

heißt es als Lebensprinzip des Menschlichen 1795/96 im Vorwort der vorletzten Fassung Hölderlins Briefroman 
Hyperion oder Der Eremit in Griechenland ${ }^{12}$ ([12, Bd. 3, S. 236]). Exzentrisch bedeutet hier abweichend von der Kreisform, mal beschleunigend, mal retardierend wie im Planetenlauf aufgrund des 2. Keplerschen Gesetzes, unregelmäßig und unvollkommen, so wie die Ellipsen in der Antike angesehen wurden, die sich aber letztlich als die mathematisch wahren Bahnen der Planeten herausstellten (vgl. [21]).

Schon 1789 beginnt Hölderlin, die kleine Maulbronner und Leonberger Welt zu vergessen, sein Streben ist auf die universitäre Bildung und die neuen, geistig anregenderen Freunde in Tübingen gerichtet - mit dem Stuttgarter Georg Friedrich Wilhelm Hegel (1770-1831) und dem jungen Leonberger Genie Friedrich Wilhelm Joseph Schelling (1775-1854), die sich beide später in ihren Naturphilosophien auf Kepler beziehen werden ([28, S. 18]), wird er sich 1790 im Stift ein Zimmer teilen. Die Verbindung zum treuen Immanuel Nast lässt er einschlafen ([24]); die noch beim Abschied in Maulbronn „unzertrennbare Liebe“ im Gedicht An Louise Nast löst er 1790 durch einen letzten Brief an sie. $^{13}$

Hölderlins Gedicht Keppler jedoch ist der Beginn der deutschen literarischen Beschäftigung mit dem Mathematiker und Astronomen jenseits der Gelegenheitsdichtung des 18. Jahrhunderts. So werden auch die nachfolgenden Tübinger Stiftler, Theologiestudenten und späteren Schriftsteller Justinus Kerner (1786-1862) 1813, Eduard Mörike 1837 und Karl Gerok (1815-1890) 1870 Kepler in Gedichten ihre Reverenz erweisen, und im Laufe des 19. Jahrhunderts kommen weitere literarische Gattungen hinzu, Romane, Erzählungen und Theaterstücke, auch über Keplers Mutter Katharina (1547-1622) und ihren Hexenprozess.

\section{Anmerkungen}

1. Hölderlins Name ist vor allem verbunden mit seinem Briefroman Hyperion oder der Eremit in Griechenland (1797/99), dem Trauerspiel Tod des Empedokles (1826) und seiner Oden- und Hymnendichtung. Zu Leben und Werk siehe [6] und [20].

2. Keplers Name ist vor allem verbunden in der Astronomie mit dem 1., 2. und 3. Keplerschen Gesetz der Planetenbewegungen (1. Die Planetenumlaufbahnen sind stets Ellipsen, in deren einem Brennpunkt die Sonne steht; 2. Die Verbindungslinie Sonne $\leftrightarrow$ Planet überstreicht während des Umlaufs um die Sonne in gleichen Zeiträumen gleich große Flächenanteile der Ellipse; 3 . Für die Umlaufzeiten $t_{1}$ und $t_{2}$ zweier Planeten und ihre mittleren Abstände $a_{1}$ und $a_{2}$ von der Sonne (große Halbachsen der Ellipsen) gilt

$$
\frac{t_{1}^{2}}{t_{2}^{2}}=\frac{a_{1}^{3}}{a_{2}^{3}}
$$

woraus für ein Planetensystem $S$ folgt

$$
\frac{t^{2}}{a^{3}}=\text { const }_{S}
$$

(analog für einen Planeten und seine Monde).) und dem Keplerschen Fernrohr, ein noch bis ca. 1900 gebräuchliches Linsenfernrohr mit zwei bikonvexen Sammellinsen, sowie in der Mathematik mit der Keplerschen Fassregel der Numerischen Integration

$$
\int_{a}^{b} f(x) d x \cong \frac{b-a}{6} \cdot\left[f(a)+4 f\left(\frac{a+b}{2}\right)+f(b)\right],
$$

ein Spezialfall (mit $m=1$ ) der späteren Simpson-Regel für $2 m+1$ Stützstellen, den Keplerschen Logarithmen mit den Funktionalgleichungen 1. $L(g)=0$,

2. $L^{\prime}(g)=-1$,
3. $L(x)+L(y)=L\left(\frac{x * y}{g}\right)$ mit $g:=10^{7}$,

so dass $x * y=g * L^{-1}(L(x)+L(y))$ analog zu den Logarithmen von John Napier (1550-1617), den Keplerschen Sternkörpern Dodekaederstern und Ikosaederstern, die zusammen mit den beiden von Louis Poinsot (1777-1859) entdeckten die vier allein möglichen regelmäßigen nichtkonvexen Körper bilden, und der Keplerschen Vermutung der Dichte unendlicher Kugelpackungen im $\mathbb{R}^{3}$, die erst 1998 durch Thomas Hales $\left({ }^{*} 195^{8}\right)$ bewiesen werden konnte. $\mathrm{Zu}$ Leben und Werk siehe $[1,2]$ oder [7].

3. Siehe $[25$, S. $33-44],[16$, S. $40-46],[6$, S. 346$]$.

4. Nach der Reformation in Württemberg eingeführt, war diese "Schwäbische Laufbahn" innovativ und führend in Deutschland, zu Hölderlins Zeiten jedoch schon etwas verstaubt und reformbedürftig. 5. Kepler war ein begeisterter und auch talentierter Dichter. Wir kennen von ihm zwei griechische, fünf deutsche und 81 lateinische Gedichte mit insgesamt 2133 Versen $([4,18])$. Am bekanntesten ist sein selbst verfasstes Epitaph „Mensus eram coelos, nunc terrae metior umbras. Mens coelestis erat, corporis umbra iacet." (Ich habe [hatte] die Himmel gemessen, nun messe ich der Erde Schatten. War des Himmels mein Geist, liegt hier des Körpers Schatten.), ein elegisches Distichon (Hexameter plus Pentameter).

6. Keplers Lehrer war der Mathematiker und Astronom Michael Mästlin (1550-1631), der ihn mit dem Werk Kopernikus' bekannt machte, Hölderlins Lehrer waren der Mathematiker und Astronom Karl Felix Seyffer (1762-1821) und vor allem der Mathematiker, Physiker und Astronom Christoph Friedrich Pfleiderer (1736-1821), der u. a. die Keplerschen Gesetze und das Newtonsche Gravitationsgesetz ausführlich behandelte ([12, Bd. 1.2 S. 555, 594], [28, S. 38-39]).

7. Die Überlieferungsgeschichte ist insofern ungewöhnlich, als die zweiseitige Handschrift Hölderlins die Zeitläufe in zwei Teilen überdauert hat; die Verse 1-24 befinden sich heute im SchillerNationalmuseum/Deutschen Literaturarchiv Marbach, der Rest - mit Echtheitsbestätigung Eduard Mörikes (1804-1875), der Hölderlin noch persönlich kannte - im Freien Deutschen Hochstift Frankfurt, und das Gedicht wurde erst posthum 1846 in der von seinem Biographen Christoph Theodor Schwab (1821-1883) herausgegebenen ersten Ausgabe sämtlicher Werke Hölderlins veröffentlicht ([11, Bd. 2, S. 167-168]). Orthographie hier gemäß [12, Bd. 1.1, S. 81], siehe auch [12, Bd. 1.2, S. $382-384]$ und $[13$, S. $72-73,540-541]$.

8. Siehe [12, Bd. 1.2, S. 383-384], [22, Bd. 4, S. 85-86], [26, S. 104105]. Die Gedenkstätten gelangten nie zur Ausführung. Schillers Text lautete

\section{IOANNES KEPPLERVS \\ FORTVNA MAIOR \\ NEVTONI \\ PER SIDERA \\ DVCTOR.}

(Johannes Kepler, durch sein Geschick hochbedeutsamer Führer für Newton zu den Gestirnen).

9. Keplers Zeitgenossen blieben ihm die verdiente wissenschaftliche Anerkennung weitgehend schuldig. Sein Nachfolger als kaiserlicher Hofmathematiker wurde 1633 der Katholik und Jesuit Athanasius Kircher (1602-1680), der in seinem Werk Magnes sive de arte magnetica opus tripartitum (1641) das Weltbild Keplers Amtsvorgängers Tycho Brahe (1546-1601) vertrat (Erde im Mittelpunkt, von Mond und Sonne umkreist, um die wiederum die restlichen Planeten laufen).

10. Beispielsweise waren 1628 beim Wechsel Keplers aus den kaiserlichen Diensten zu Herzog Albrecht von Wallenstein (1583-1634) noch 11817 Gulden Besoldung offen ( 960 ooo $€$ nach heutigem Wert, an Kaufkraft vermutlich das Vierfache). Seine Witwe Susanna (1589-1636) bekam 1633 eine Obligation der kaiserlichen Hofbuchhaltung über 12694 Gulden und 13 Kreuzer (incl. Zinsen), die jedoch von den Nachkommen nie eingelöst werden konnte.

11. Pikanterweise gestaltet nach einem Kepler-Gemälde, das um 1810 in Regensburg gefunden und Anfang des 20. Jahrhunderts als falsch erkannt wurde; es zeigt in Wirklichkeit Herzog Ludwig X. von BayernLandshut (1495-1545) ([14,29]).

12. Hyperion, „der oben Gehende, der in der Höhe Wandelnde“, in der griechischen Mythologie einer der Titanen, Sohn von Uranos (Himmel) und Gaia (Erde); auch Name des 1848 entdeckten achten Saturnmondes.

13. Siehe $[12$, Bd. 1.1, S. 64 , Bd. 1.2, S. $371-372$, Bd. 6.1, S. 50-52], $[26$, S. 24]. 


\section{Literatur}

[1] Albinus, Hans-Joachim: Joannes Keplerus Leomontanus. Kepler's Childhood in Weil der Stadt and Leonberg 1571-1584. The Mathematical Intelligencer 24 (2002), Nr. 3, S. 50-58.

[2] Albinus, Hans-Joachim: Johannes Kepler im und nahe dem alten Herzogtum Württemberg. Vom Leonberger Lateinschüler zu einem der Begründer der modernen Astronomie - zum 444. Geburtstag. Schwäbische Heimat 66 (2015), S. 459-467.

[3] Albinus, Hans-Joachim: Erforscher des Schöpfungsplans. Zum 444. Geburtstag ein Blick auf das Leben des Astronomen, Mathematikers, Physikers und gläubigen Protestanten Johannes Kepler. Stuttgarter Zeitung 71 (2015), Nr. 297 (23. 12. 2015), S. 24. tinyurl.com/y6x44jtq

[4] Albinus, Hans-Joachim: In memoriam Joannis Kepleri poetae. Selbst wer mit Johannes Keplers Leben vertraut ist, weiß meist nicht: Der große Astronom war auch Poet. Jetzt sind seine Gedichte publiziert. Leonberger Kreiszeitung 75 (2019), Nr. 93 (20.4. 2019), S. VII tinyurl.com/y2xo2rr6

[5] Becher-Hedenus, Doris: „Wir durchlaufen alle eine exzentrische Bahn“. Die deutsche Kepler-Rezeption im 18. Jahrhundert und das Regensburger Denkmal von 1808. Regensburger Studien und Quellen zur Kulturgeschichte 19. Universitätsverlag, Regensburg, 2010.

[6] Beck, Adolf; Raabe, Paul (Hrsg.): Hölderlin. Eine Chronik in Text und Bild. Schriften der Hölderlin-Gesellschaft 6-7. Insel, Frankfurt, 1970.

[7] Caspar, Max: Johannes Kepler. Verlag für Geschichte der Naturwissenschaften und der Technik, Stuttgart, 2019, 5. Aufl.

[8] Drecoll, Volker Henning; Baur, Juliane; Schöllkopf, Wolfgang (Hrsg.): Stiftsköpfe. Mohr Siebeck, Tübingen, 2012.

[9] Franz, Michael: Das Höhere Bildungswesen des Herzogtums Württemberg im 18. Jahrhundert. Franz, Michael; Jacobs, Wilhelm G. (Hrsg.): Materialien zum bildungsgeschichtlichen Hintergrund von Hölderlin, Hegel und Schelling 1 („... so hat mir / Das Kloster etwas genüzet“. Hölderlins und Schellings Schulbildung in der Nürtinger Lateinschule und den württembergischen Klosterschulen). Schriften der Hölderlin-Gesellschaft 23/1. Isele, Eggingen, 2004, S. 11-36.

[10] Franz, Michael: Das Magisterstudium der Philosophie in Tübingen um 179o. Franz, Michael (Hrsg.): Materialien zum bildungsgeschichtlichen Hintergrund von Hölderlin, Hegel und Schelling 2 („... im Reiche des Wissens cavalieremente“? Hölderlins, Hegels und Schellings Philosophiestudium an der Universität Tübingen). Schriften der Hölderlin-Gesellschaft 23/2. Isele, Eggingen, 2005, S. 11-23.

[11] Hölderlin, Friedrich: Sämmtliche Werke. Hrsg. von Christoph Theodor Schwab. Cotta, Stuttgart, 1846.

[12] Hölderlin, Friedrich: Sämtliche Werke. Grosse Stuttgarter Ausgabe. Hrsg. von Friedrich Beissner/Adolf Beck. Kohlhammer, Stuttgart, 1943-1985 (elektronischer Zugang über www.wlbstuttgart.de).
[13] Hölderlin, Friedrich: Sämtliche Gedichte. Hrsg. von Jochen Schmidt. Deutscher Klassiker Verlag, Frankfurt, 2005.

[14] Johann Keppler Kaiserlicher Mathematiker. Denkschrift des historischen Vereins der Oberpfalz und von Regensburg auf die Feier seines zehnjährigen Bestandes. Mit Keppler's Bildnis, Wappen und dem Faksimile seiner Handschrift. Demmler, Regensburg, 1842.

[15] Kästner, Abraham Gotthelf: Geschichte der Mathematik seit der Wiederherstellung der Wissenschaften bis an das Ende des achtzehnten Jahrhunderts. Geschichte der Künste und Wissenschaften seit der Wiederherstellung derselben bis an das Ende des achtzehnten Jahrhunderts, Abth. 7.1. Rosenbusch, Göttingen, 1796-180o (Nachdruck Olms, Hildesheim, 1970)

[16] Keicher, Ulrich: Schlummert sanft ihr modernde Gebeine. Leonberger Literaturgeschichte vom Mittelalter bis heute. Beiträge zur Stadtgeschichte 2. Stadtarchiv, Leonberg, 1994.

[17] Kepler, Johannes: Gesammelte Werke. Hrsg. von Max Caspar u. a. Beck, München, 1937-2017. kepler.badw.de/kepler-digital.html.

[18] Kepler, Johannes: Sämtliche Gedichte. Hrsg. und kommentiert von Friedrich Seck. Übersetzt von Monika Balzert. Spudasmata 180. Olms, Hildesheim, 2018.

[19] Müller, Ernst; Haering, Theodor; Haering, Hermann: Stiftsköpfe. Schwäbische Ahnen des deutschen Geistes aus dem Tübinger Stift. Salzer, Heilbronn, 1938.

[20] Safranski, Rüdiger: Hölderlin. Komm! ins Offene, Freund! Biographie. Hanser, München, 2019.

[21] Schadewaldt, Wolfgang: Das Bild der exzentrischen Bahn bei Hölderlin. Hölderlin-Jahrbuch 6 (1952), S. 1-16.

[22] Schiller, Friedrich: Nachlese zu Schillers Werken nebst Variantensammlung. Aus seinem Nachlaß im Einverständnis und unter Mitwirkung der Familie Schillers hrsg. von Karl Hoffmeister. Supplemente zu Schillers Werken, 1. Cotta, Stuttgart, 1840-1841.

[23] Schmidt-Kaler, Theodor: Hölderlin und die Astronomie. Beiträge zur Astronomiegeschichte 2 (1999, 2. Aufl. 2002), S. 122-127.

[24] Schönwitz, Ute: Immanuel Nast schreibt einen Brief, aber Hölderlin antwortet nicht. Eine Spurensuche. Radius, Stuttgart, 2005.

[25] Schönwitz, Ute: Ich bin sehr schön logiert. Literarische Geschichten aus Leonberg. Warmbronner Anstöße 3. Warmbronn, 2011.

[26] Siegmund-Schultze, Friedrich: Der junge Hölderlin. Analytischer Versuch über sein Leben und Dichten bis zum Schluß des ersten Tübinger Jahres. Sprache und Kultur der germanischen und romanischen Völker, B (Germanische Reihe) 32. Priebatsch, Breslau, 1939.

[27] Volke, Werner: Hölderlin in Tübingen. Marbacher Magazin 11. Deutsche Schillergesellschaft, Marbach, 2001, 4. Aufl.

[28] Ziche, Paul; Rezvykh, Petr: Sygkepleriazein. Schelling und die Kepler-Rezeption im 19. Jahrhundert. Schellingiana 21. FrommannHolzboog, Stuttgart, 2013.

[29] Zinner, Ernst: Die Kepler-Bildnisse. Karl Stöckl (Hrsg.): KeplerFestschrift. Bd. I. Berichte des Naturwissenschaftlichen Vereins zu Regensburg 29 (1928/1930), S. 337-346.

Hans-Joachim Albinus war von 1992 bis 2020 Ministerialrat im Innenministerium Baden-Württemberg, zuletzt als Leiter des Inneren Dienstes, nun im Ruhestand. Er studierte Mathematik und Geographie an der Ruhr-Universität Bochum und Informatik an der FernUniversität Hagen. Neben dem Beruf beschäftigte er sich mit Geschichte der Mathematik, speziell mit Johannes Kepler, und ist Mitglied der Kepler-Gesellschaft. Er war einer der „Mathemacher" des Wissenschaftsjahres 2008 und initiierte die jährliche gemeinsame Kepler-Veranstaltungsreihe der Kepler-Städte Weil der Stadt und Leonberg, wo er auch ehrenamtlich Stadtführungen speziell zu Kepler anbietet. 